\title{
Localized reversal of the perpendicular velocity in Tore Supra ohmic, L-mode, limited plasmas
}

\author{
E. $\operatorname{Trier}^{1 \dagger}$, P. Hennequin ${ }^{1}$, Ö. D. Gürcan ${ }^{1}$, R. Sabot ${ }^{2}$, J. Bucalossi $^{2}$, Z.O. Guimarães-Filho ${ }^{2 \ddagger}$, C. Bourdelle ${ }^{2}$, \\ F. Clairet ${ }^{2}$, G. Falchetto ${ }^{2}$, C. Fenzi ${ }^{2}$, X. Garbet ${ }^{2}$, P. Maget $^{2}$, L. Vermare ${ }^{1}$ and the Tore Supra team
}

${ }^{1}$ Laboratoire de Physique des Plasmas, Ecole Polytechnique, CNRS, F-91128 Palaiseau Cedex

${ }^{2}$ CEA, IRFM, F-13108 Saint Paul-lez-Durance, France

\begin{abstract}
In Tore Supra plasmas, the perpendicular velocity measured by Doppler reflectometry was observed to reverse in a localized zone close to a normalized radius $\sim 0.5-0.6$, changing from a negative value (corresponding to a negative radial electric field $\left.E_{r}\right)$ to a positive value $\left(E_{r}>0\right)$. This occurs in L-mode, ohmic plasmas with a negligible external momentum input, a non-circular limited cross-section, and an edge safety factor close to 3 . This reversal is favoured by a decrease in the magnetic field, or an increase in density. It is accompanied by a characteristic behaviour of the MHD fluctuation RMS signal, whose amplitude decrease during a ramp-down of the edge safety factor as it approaches $q_{a} \sim 3.1-3.2$. A $m / n=2 / 1$ mode is involved in the mechanism causing these observations.
\end{abstract}

\section{Introduction}

Understanding the heat and particle transport in tokamaks requires a quantitative knowledge of the mechanisms enforcing the value of the radial electric field $\left(E_{r}\right)$, whose radial shear plays a crucial role in the formation of transport barriers [1,2,3]. External momentum sources like Neutral Beam Injection are one of the main drive for toroidal rotation, and therefore $E_{r}$ which is closely related to the plasma toroidal velocity $\left(V_{\Phi}\right)$ through the radial force balance equation. However, fusion reactors are likely to receive negligible external momentum input, which raises interest in studying spontaneous toroidal rotation and associated $E_{r}$.

The radial electric field evolution equation has the form $\partial E_{r} / \partial t \propto \sum e_{k} \Gamma_{k}$, where $\Gamma_{k}$ are the surfaceaveraged radial fluxes of the species of charge $e_{k}$ (e.g. [4]). A theoretical difficulty for the knowledge of mechanisms determining $E_{r}$ comes from the automatic ambipolarity of the collisional transport in a perfectly axisymmetric plasma [5]. Thus, only turbulent fluxes (which are mostly ambipolar) or fluxes related to a loss of axisymmetry are expected to have an effect on $E_{r}$. Such a non-axisymmetry can for example arise from MHD perturbations, or from the toroidal field ripple, which is a modulation of the toroidal magnetic field amplitude due to the discrete number of coils.

In Tore Supra, the source of external momentum is not strong, and the ripple is large: $\delta=\left(B_{\max }-\right.$ $\left.B_{\min }\right) /\left(B_{\max }+B_{\min }\right)$ extends up to $7 \%$. Previous comparisons [6] between $E_{r}$ measurements by Doppler reflectometry and predictions of $E_{r}$ due to ripple-induced non ambipolar diffusion have shown a good agreement in the zone $0.6<r / a<0.8$. $E_{r}$ predicted value is negative to compensate ion losses that would be dominant in the absence of an ambipolar radial electric field $[7,8,9]$. Such a negative value is observed routinely by Doppler reflectometry in the part of the confinement region that is accessible (typically $0.5 \lesssim r / a<1$ ).

In this article, observations of a localized reversal of $E_{r}$ in Tore Supra are reported, which occurs in plasma conditions unusual for this machine, at low magnetic field and safety factor, accompanied with significant

\footnotetext{
${ }^{\dagger}$ Current address: Max Planck Institute for Plasma Physics, D-85748 Garching, Germany

$\ddagger$ Current address: Institute of physics, University of São Paulo, 05508-090 São Paulo, São Paulo
} 
MHD activity. The interest of these observations is to show the existence of another mechanism that is strong enough to compete with the otherwise dominant ripple-induced particle fluxes. Experiments have been carried out to study the sensibility of this reversal with main plasma parameters.

The paper is organized as follows: in a first part, Doppler reflectometry, used for radial electric field evaluation, is briefly described. The second section presents the observations and the investigation of its parametric dependence with main plasma parameters. Complementary measurements aiming at investigating the mechanism causing this radial electric field reversal are in section 3, before a discussion in section 4 .

\section{Plasma conditions and diagnostics}

\section{$1.1 \quad$ Tore Supra}

In Tore Supra, a tokamak in limited configuration with a nominal major and minor radius of $R=2.35 \mathrm{~m}$ and $a=0.72 \mathrm{~m}$ respectively, the magnetic field $B_{\phi}$ can be set up to $4 \mathrm{~T}$ and the plasma current $I_{p}$ to 1.5 MA. Tore Supra is usually operated with a vacuum magnetic field $B_{0}>3 \mathrm{~T}$ and a circular cross-section.

In the experiments presented in this paper, plasma parameters domain is relatively far from Tore Supra usual conditions: $B_{\phi}=1.9 \mathrm{~T}, I_{p} \approx 745 \mathrm{kA}$, edge safety factor $q_{a}$ close to 3 , and a non-circular plasma cross section. All the plasmas considered in this paper are ohmic, L-mode, and have negligible external momentum input.

\subsection{Doppler reflectometry}

Doppler reflectometry $[10,11]$ is based on the backscattering of an incident microwave beam by the density fluctuations whose wave-number $\left(\mathbf{k}_{f}\right)$ matches a Bragg rule with respect to the incident wave-number $\left(\mathbf{k}_{i}\right)$ $: \mathbf{k}_{f}=-2 \mathbf{k}_{i}$. The beam is launched perpendicular to the magnetic field lines, towards the plasma centre, with a variable poloidal angle for selecting the local wave number. The backscattered signal comes mainly from the cut-off area, whose location is determined by the probing frequency; all the measurements presented here are in O-mode polarization. The backscattered signal is Doppler-shifted $\Delta \omega \simeq k_{f, \perp} V_{f, \perp}$, where $V_{f, \perp}=$ $v_{E \times B}+v_{f, f l u c}$ is the perpendicular velocity of density fluctuations of wave-number $\mathbf{k}_{f}, v_{E \times B}$ is the electric drift, $V_{f, \perp}\left(\right.$ resp. $v_{f, f l u c}$ ) are the perpendicular velocity (resp. phase velocity) of these fluctuations (the $f$ index will be omitted). The term $v_{f l u c}$ is often assumed to be negligible in comparison with $v_{E \times B}$ [12]: this yields $V_{\perp} \approx v_{E \times B}=E_{r} /|B|$. If the phase velocity was not negligible (as alredy observed in Tore Supra [13]), this would result in a radially-extended shift of the $V_{\perp}$ profile, rather than a localized abrupt change. The localized reversal of the perpendicular velocity presented in this article is therefore attributed to a change in $E_{r}$. Note that with the chosen convention $V_{\perp}>0$ for a flow in the ion diamagnetic direction. In the plasmas considered here, the acquisition scheme is discontinous in time: $V_{\perp}$ radial profiles are measured every 450 $\mathrm{ms}$, each profile consisting in 10 radial positions (typically lying in the range $r / a \sim 0.5-0.7$ ) acquired by a scan of the probing frequency in the range $49-60 \mathrm{GHz}$, with $5 \mathrm{~ms}-$ long frequency steps.

The signal is formed from contributions all along the beam, with a dominant one expected to be backscattered close to the cut-off, where the wave-number is mainly poloidal: this results in a a significant Dopplershifted component $[11,14]$. Other effects such as forward scattering in the cut-off region, or backscattering on strong fluctuations close to the separatrix, lead to another narrower component centered around $f=0$ since the wave number in such cases is small or mainly radial. In this study, the Doppler component has a relatively low amplitude compared to this unshifted component (see an example in figure 1) which makes it difficult to separate their respective contributions. This is due to the low density, and to the non-normal incidence angle when the cut-off approaches mid-radius, i.e. the limits of the accessible zone. Observations will be presented under the form of raw spectra for qualitative analysis. A crude quantification of the Doppler shift will however be obtained from a simple method for separating the Doppler and unshifted components, using the fact that the unshifted component is quasi symmetrical relatively to the $f=0$ axis. The Doppler component, provided its shift is large in comparison with its half-width, is then approximately twice the positive part of the odd component of a spectrum $S(f)$ : Doppler $(f) \approx \operatorname{odd}(S(f))+|\operatorname{odd}(S(f))|$. In practice, these assumptions are realistic enough to allow a good extraction of the Doppler component provided the frequency shift is sufficiently large $(\gtrsim 150 \mathrm{kHz})$. For the sake of visualization, this decomposition can also be applied on a logarithmic spectra. This will be done in figure 4 and is useful for a approximate display of the 
Doppler component - however in this case Doppler $r_{l o g} \neq \operatorname{odd}\left(S_{l o g}\right)+\left|\operatorname{odd}\left(S_{l o g}\right)\right|$ and a typical shift of $20 \%$ will remain between this apparent "logarithmic" Doppler shift and the real one (since the study is qualitative and the observed phenomenon consists in a reversal of the perpendicular velocity, this magnitude of error can be considered as acceptable). The following rough estimator of the dominant Doppler frequency will also be used: $\left\langle f_{\text {Doppler }}\right\rangle \equiv \int\left(f . S_{\text {asym. }, \text { log }}\right) d f / \int S_{\text {asym. }, \text { log }} d f$ where $S_{\text {asym. }, \log } \equiv \operatorname{odd}\left(\log _{10} S(f)\right)+\mid$ odd $\left(\log _{10} S(f)\right) \mid$.

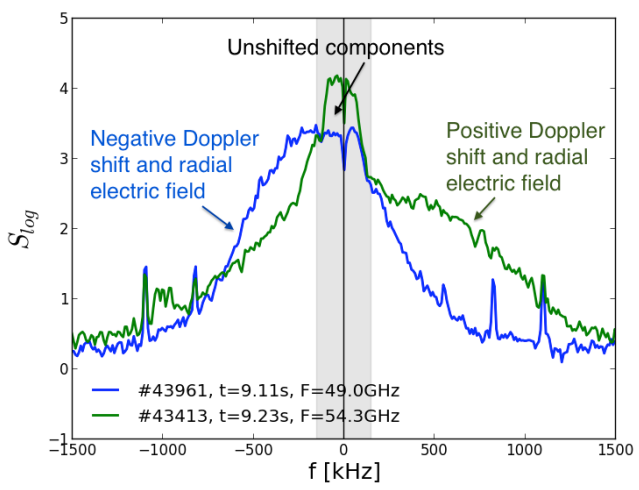

Figure 1: Examples of Doppler spectra with a negative (blue) and positive (green) Doppler shift.

\section{Perpendicular velocity reversal, and its dependence to plasma parameters}

The methodology followed in this study is to vary the plasma parameters around a reference plasma state, which corresponds to a clear observation of the perpendicular velocity reversal. A database of 18 discharges has been gathered. The perpendicular velocity reversal showed a good reproducibility. It was observed to be sensitive to small variation of plasma parameters. The effect of the edge safety factor $q_{a}$, the toroidal vacuum magnetic field $B_{0}$, and the plasma density have been investigated.

\subsection{Influence of the edge safety factor}

In this experimental database, the discharge \#43413 (figure 2a) which is one of the clearest early observation of the $V_{\perp}$ reversal, is used as reference. It consists in a current ramp from 600 to $745 \mathrm{kA}$ in a ohmic plasma with a moderate density (central line-integrated density $n_{l}=3.3-3.6 \times 10^{19} \mathrm{~m}^{-2}$ ), and a non-circular cross-section (ellipticity $b / a \simeq 1.15$, triangularity $\delta \simeq 0.2$ ). The edge safety factor $q_{a}$ is decreased from 3.6 to 2.8. Several $V_{\perp}$ profiles are acquired at various times of the $I_{p}$ ramp (such an acquisition scheme is applied to every considered discharges).

The figure $2 \mathrm{~b}$ displays Doppler reflectometry spectra at different times of the decreasing $q_{a}$ ramp, and at a constant probing frequency of $53.4 \mathrm{GHz}$ (which observes the $r / a \sim 0.5-0.6$ region, and fluctuations with a wave-number $10.5<k_{\perp}<12 \mathrm{~cm}^{-1}$ ). In the chosen convention, $V_{\perp}$ and $E_{r} \simeq|B| V_{\perp}$ have the same sign. At the beginning of the discharge, when $q=3.45$, no Doppler-shifted component is detectable. There are two possible (and compatible) explanations for this: one is that $\left|V_{\perp}\right|$ is so weak that the Doppler component cannot be distinguished from the unshifted $f=0$ component, the other is that a Doppler shift exists but that the corresponding component amplitude is too low to be detectable. But while $q_{a}$ is decreased, positive $V_{\perp}$ appears progressively, even before the crossing of the LCFS by $q=3$ (e.g. it is clear when $q_{a}=3.07$ ). At the end of the discharge, $q_{a}<3$ and the perpendicular velocity at the reversal is of the order $2-3 \mathrm{~km} / \mathrm{s}$. The presence of this $V_{\perp}>0$ component is then observed at two probing frequencies of the incident beam (i.e. 

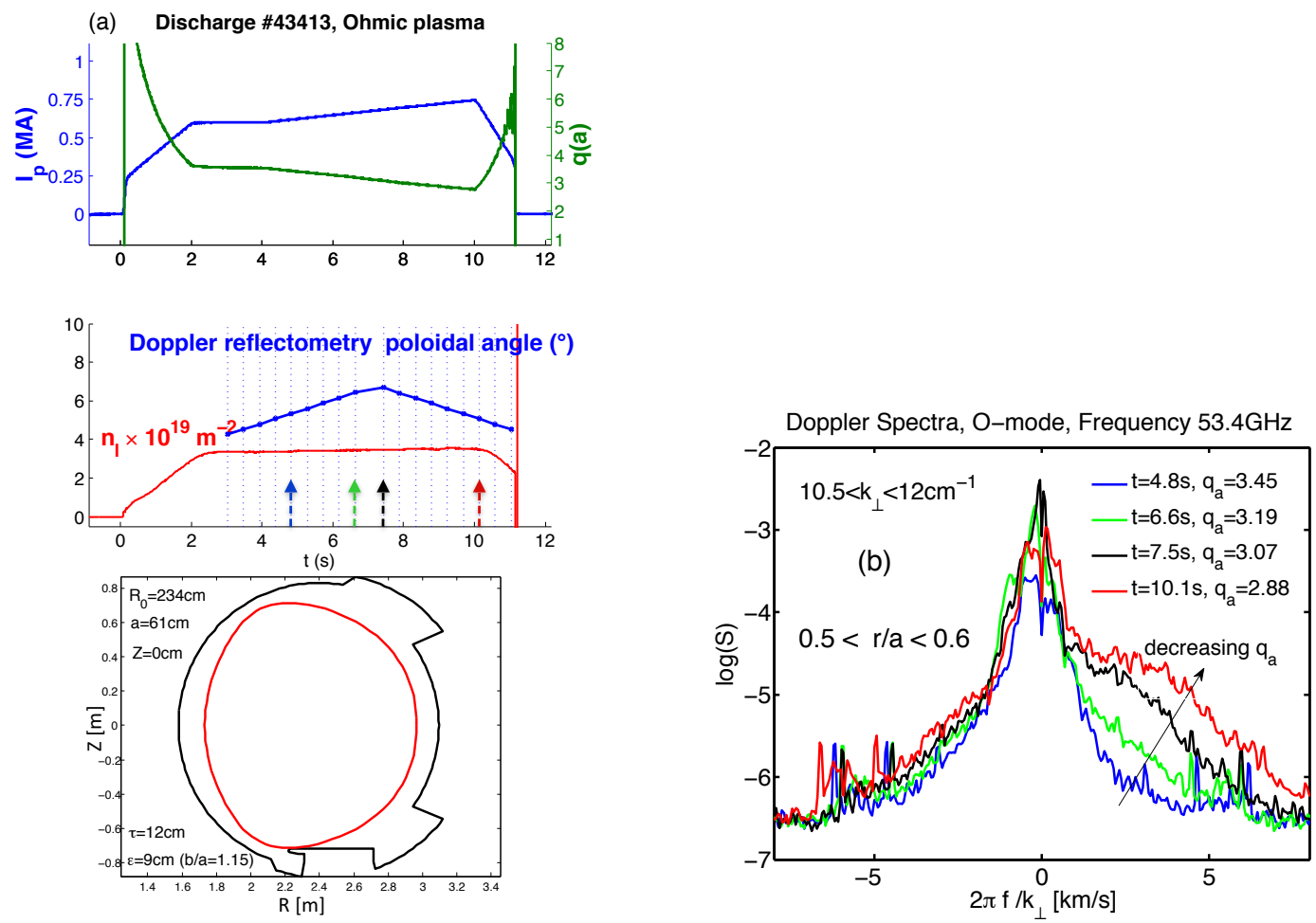

Figure 2: (a) Scenario for discharge \#43413, showing the decreasing ramp of the edge safety factor (upper panel), the central line-integrated electron density, the time of Doppler measurements (vertical dotted lines, lower panel), and the plasma cross-section. (b) Doppler spectra measured in O-mode at 53.4 GHz, at times symbolized by colored arrows in subfigure a. Measurement localization depends on density and hence changes with time, but lies in the $r / a \sim 0.5-0.6$ range. In $x$-axis, the Doppler shift frequency is converted to a perpendicular velocity $V_{\perp}=\Delta \omega / k_{\perp}$. In addition to the central component centered on $f=0$, always present, a Doppler component corresponding to a positive $E_{r}$ emerges when $q_{a}$ decreases. 
radial positions), which provides an estimate for the spatial extent of the reversal region: $\triangle(r / a) \sim 0.05-0.07$, hence an approximate width of $3-4 \mathrm{~cm}$.

It is also interesting to compare the $V_{\perp}$ evolution in the discharge \#43413 with the corresponding evolution in the discharge \#43961, where no reversal was observed. The latter shot consists in a circular plasma, a decreasing $q_{a}$ ramp from 4 to 3.1, slightly higher density and magnetic field $(1.97 \mathrm{~T}$ instead of $1.9 \mathrm{~T})$ : see figure 3. As discussed below, magnetic field, density and plasma shape are parameters that influence the $V_{\perp}$ reversal. The compared evolutions of $V_{\perp}$ profiles are displayed in figure 4 . This figure shows the asymmetric part of the $V_{\perp}$ logarithmic spectra decomposed according to the method described in section 1.2: this provides an estimate for the Doppler-shifted component. For the shot \#43413 (but not for \#43961), a positive $V_{\perp}$ is detected in a localized zone, close to $r / a=0.5-0.55$, that do not extend up to the plasma centre (even if measurements at smaller $r / a$ are difficult, this can be noticed).

This discharge \#43413 (and the other 3-4 similar discharges from this series of experiments, where the same behaviour is observed) shows the crucial role that plays the edge safety factor $q_{a}$ in the $V_{\perp}$ reversal.
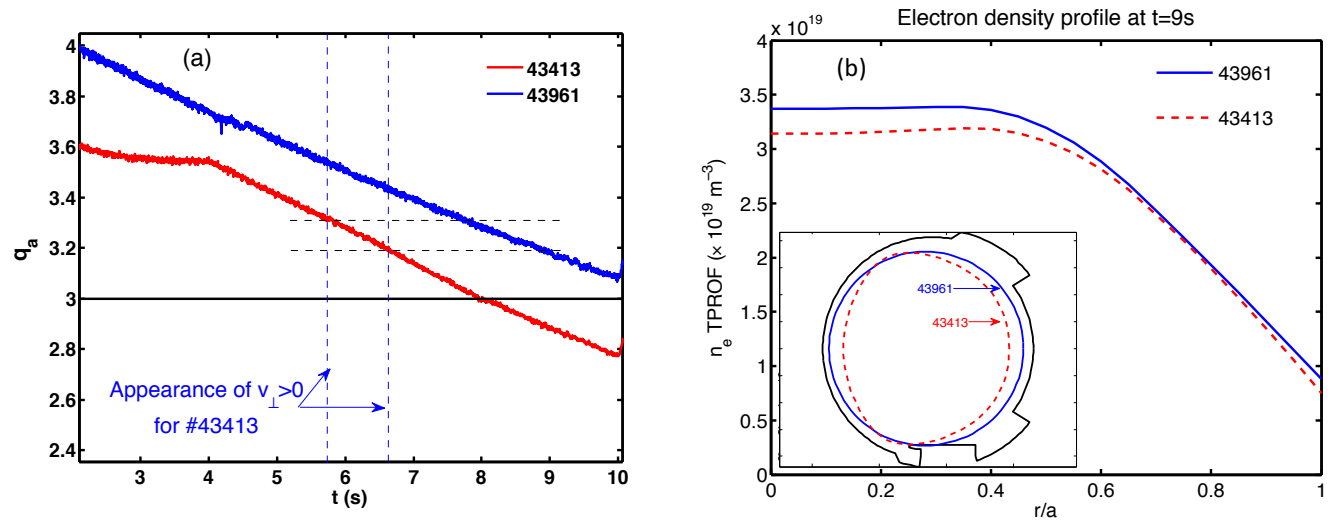

Figure 3: (a) Time evolution of edge safety factor $q_{a}$ during shots \#43413 and \#43961. The time interval corresponding to the appearance of the reversal for \#43413 and the associated $q_{a}$ are represented in dashed lines. (b) Electron density profiles reconstructed from interferometry and Thomson scattering measurements, at $t=9$ s, shown with plasma sections. 

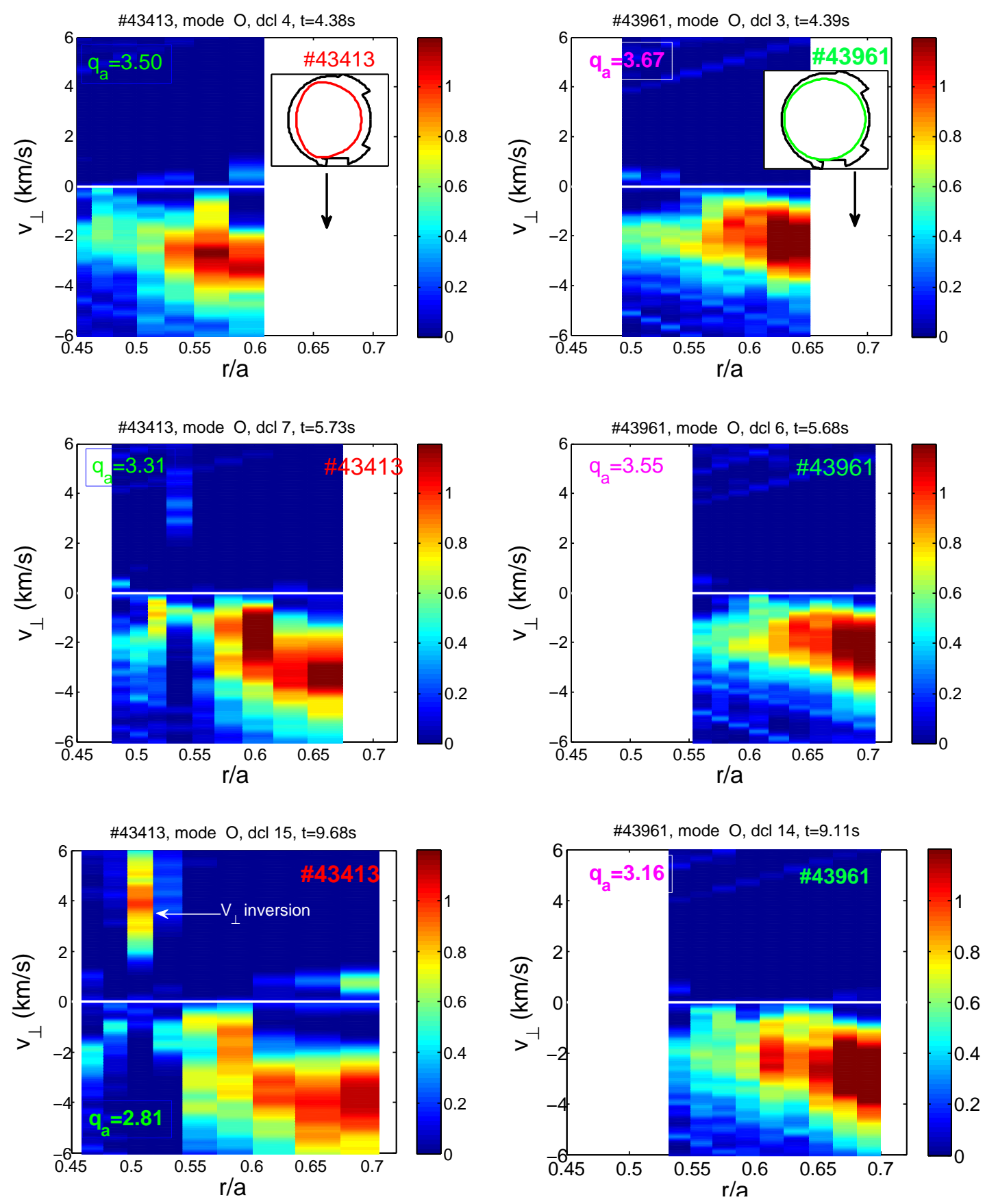

Figure 4: Spectrograms representing the Doppler component strength (quantified by odd $\left(\log _{10} S\right)+$ $\left|\operatorname{odd}\left(\log _{10} S\right)\right|$, cf. section 1.2), for shots \#43413 ( $V_{\perp}$ reversal) and \#43961 (no detected reversal). In y-axis, the Doppler frequency shift is multiplied by $2 \pi / k_{\perp}$, thus converted to a perpendicular velocity. 


\subsection{Influence of plasma density}

\begin{tabular}{|c|c|c|c|c|c|}
\hline Shot & $B_{0}(\mathrm{~T})$ & $n_{l}^{(4)} \times 10^{19} m^{-2}$ & $I_{p}(k A)$ & $q_{a}$ & Scenario \\
\hline $43413($ ref $)$ & 1.90 & $3.3-3.6$ & $600-745$ & $3.6-2.80$ & $q_{a}$ ramp-down \\
\hline 43950 & 1.93 & $3.0-3.4$ & $600-745$ & $3.6-2.85$ & $q_{a}$ ramp-down \\
\hline 43951 & 1.93 & $3.3-3.7$ & $600-765$ & $3.6-2.75$ & $q_{a}$ ramp-down \\
\hline 43952 & 1.93 & $3.3-3.9$ & 700 & $3.03-2.98$ & density ramp-up \\
\hline 43959 & 2.02 & $3.4-4.1$ & 820 & $2.8-2.7$ & density ramp-up \\
\hline
\end{tabular}

Table 1: Plasma main characteristics of discharges showing the effect of electron density on the $V_{\perp}$ reversal (in the form: starting value-end value). Line integrated density $n_{l}$ is measured on a central chord ( $\left.n^{\circ} 4\right)$. Plasma sections are identical to \#43413 (figure 3).

The effect of plasma electron density on the $V_{\perp}$ reversal can be shown by considering the discharges from table 1. The first couple of compared discharges is \#43950 and \#43951, which are similar to reference \#43413: same plasma shape (as in figure 3), but slightly higher magnetic field (1.93 T instead of $1.90 \mathrm{~T}$ ). As shown in figure 5a, density is lower for \#43950 than for \#43951 and \#43413. The figure 5b compares the Doppler spectra whose, among all spectra acquired in these shots (i.e. including all time steps and Doppler probing frequencies), have the largest energy fraction in the $V_{\perp}>0$ domain. A $V_{\perp}$ reversal can be detected for $\# 43951$ whereas it is not the case for $\# 43950$, which has a lower density $\left(\Delta n_{l} \approx 0.3 \times 10^{19} \mathrm{~m}^{-2}\right)$.

The effect of a lower density on Doppler measurements should be the following: (1) a displacement of the cutoff layer towards the plasma centre, and (2) a decrease of the spectrum energy (because signal is proportional to the scattering source density). The cut-off layer displacement should not prevent from observing a reversal, since the zone of accessible radius for measurements is extended. The decrease of the spectrum energy associated with positive $V_{\perp}$ from \#43951 to \#43950 is too large to be explained by the $10 \%$ relative variation of plasma density: the absence of the $V_{\perp}$ reversal at lower density is therefore not related with diagnostic accessibility issues.
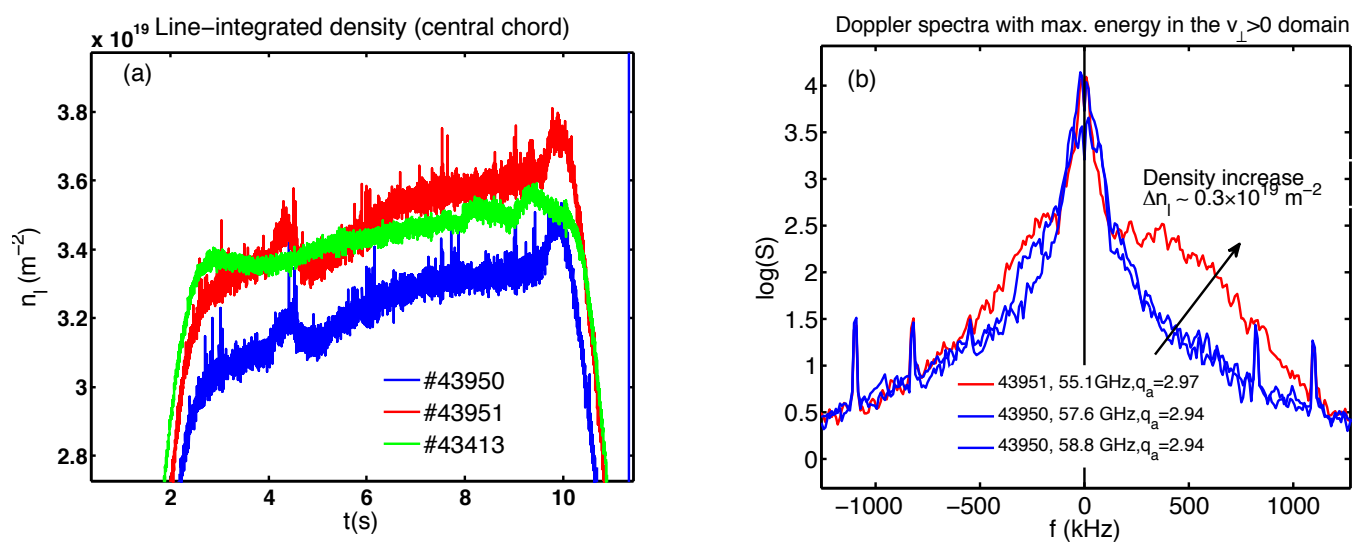

Figure 5: (a) Line-integrated electron density (central chord) for discharges \#43950, \#43951, and reference \#43413. (b) Doppler Spectra with the largest energy fraction in the $f>0$ (i.e. $V_{\perp}>0$ ) domain, for shot \#43950 and \#43951.

A second illustration of the influence of plasma density are the discharges \#43952 and \#43959, for which a density ramp at constant $q_{a}$ is done. The $q_{a}$ values (respectively 3 and 2.75) are low enough to see a reversal in the reference discharge \#43413. The line-integrated electron density and the central density reconstructed from interferometry and Thomson scattering are displayed in figures $6 \mathrm{a}-6 \mathrm{~b}$, and the Doppler spectra at the probing frequencies corresponding to the $V_{\perp}$ reversal detection are shown in figures $6 \mathrm{c}$ and $6 \mathrm{~d}$. 
The appearance of positive $V_{\perp}$ when $n_{e}$ is increased can be observed. The smallness of density variations influencing the $V_{\perp}$ reversal (10-20\%) indicates the existence of a threshold in $n_{e}$ above which it is triggered.
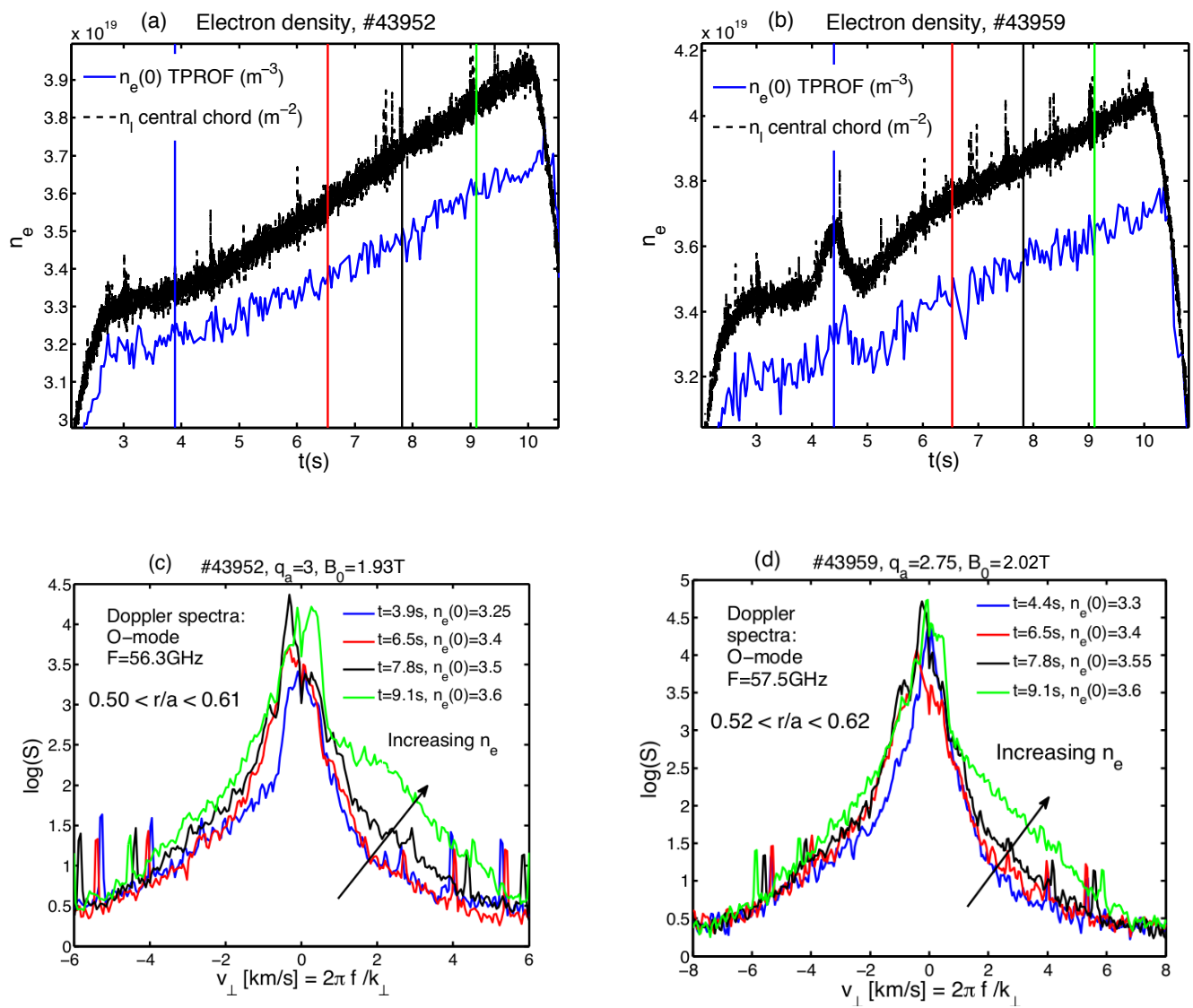

Figure 6: (a)-(b): Electron density during discharges \#43952 and \#43959: line-integrated on a central chord (black), evaluated at the plasma centre from interferometry and Thomson measurements (blue). Vertical lines indicate the times of the Doppler spectra shown in the subfigures $c$ and $d$, with the corresponding color code. (c)-(d): Evolution of the Doppler spectra, at the probing frequencies corresponding to the $V_{\perp}$ reversal detection (56.3 and $57.5 \mathrm{GHz}$ respectively), during the $n_{e}$ ramp.

\subsection{Influence of the magnetic field}

\begin{tabular}{|c|c|c|c|c|c|}
\hline & $B_{0}(\mathrm{~T})$ & $n_{l}^{(4)} \times 10^{19} m^{-2}$ & $I_{p}(k A)$ & $q_{a}$ & Scenario \\
\hline 43413 & 1.90 & $3.3-3.6$ & $600-745$ & $3.6-2.8$ & $q_{a}$ ramp-down \\
\hline 43960 & 1.97 & $3.5-3.8$ & $620-805$ & $3.6-2.7$ & $q_{a}$ ramp-down \\
\hline 43958 & 2.02 & $3.5-3.7$ & $640-815$ & $3.6-2.7$ & $q_{a}$ ramp-down \\
\hline 43957 & 2.17 & $3.8-4.1$ & $680-860$ & $3.6-2.8$ & $q_{a}$ ramp-down \\
\hline 45012 & 2.62 & 4.5 & $830-1020$ & $3.7-2.8$ & $q_{a}$ ramp-down \\
\hline 45014 & 2.62 & 4.5 & $830-1020$ & $3.7-2.8$ & $q_{a}$ ramp-down \\
\hline
\end{tabular}

Table 2: Plasma discharges showing the influence of the vacuum magnetic field $B_{0}$ on the $V_{\perp}$ reversal. Shots \#45012 and \#45014 were done in order to allow ECE measurements in the region of the reversal (see section 3). 
The influence of the vacuum magnetic field $B_{0}$ on the reversal is shown by considering discharges \#43413, \#43960 and \#43958 from table 2 . The same $q_{a}$ decreasing ramp is done for all of these discharges. The increase in $B_{0}$ from $1.90 \mathrm{~T}$ to $2.02 \mathrm{~T}$ consists in a relative variation of only $6 \%$.

Decreasing the magnetic field favours the $V_{\perp}$ reversal: this is shown in figure 7 which displays for each of these discharges the Doppler spectra for which $\left\langle f_{\text {Doppler }}\right\rangle$ is maximum. At an intermediate magnetic field of $1.97 \mathrm{~T}$, a slight appearance of a positively Doppler-shifted component can be noticed, even if $\left\langle f_{\text {Doppler }}\right\rangle<0$ for this spectrum. The smallness of the relative variation $\delta B_{0} / B_{0}<6 \%$ sufficient to induce a significant change in the spectrum shape once again reveals the presence of a threshold in $B_{0}$. For shot \#43413, the effect of a lower $B_{0}$ (favourable for the reversal) dominates the effect of a lower density (see figure 7a, unfavourable for the reversal as shown in the previous paragraph). We also point out that other discharges were done at a higher magnetic field $2.33 \leq B_{0} \leq 2.62 \mathrm{~T}$, for which no reversal was observed by Doppler reflectometry: however this could also be due to the higher density that shifts the Doppler measurement zone towards the plasma edge and out of the reversal region.
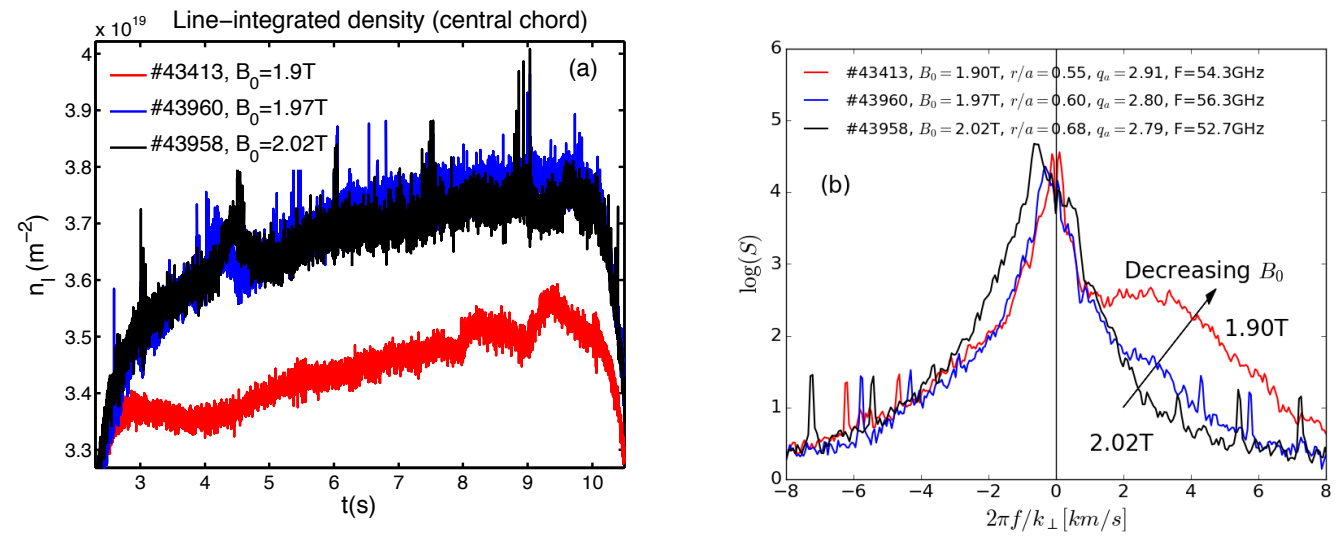

Figure 7: (a) Line-integrated density (central chord) for discharges $43413\left(B_{0}=1.90 \mathrm{~T}\right)$, $43960(1.97 \mathrm{~T})$, and $43960(2.02 \mathrm{~T})$. (b) Doppler spectra with maximum $\left\langle f_{\text {Doppler }}\right\rangle$, for each of these discharges.

\subsection{Summary of observed parameter dependences}

An overview of the $V_{\perp}$ reversal detections and their location in the $q_{a}$-density plane is presented in figures $8 \mathrm{a}$ and $8 \mathrm{~b}$. The volume-averaged density $\left\langle n_{e}\right\rangle$ is used in these plots, which is estimated from the 10 interferometry lines. These two figures take into account all the Doppler reflectometry measurements done during the discharges at lower $B_{0}$ (figure 8a, $1.90 \leq B_{0} \leq 1.93 \mathrm{~T}$, discharges \#43410-13, 43950-52) and higher $B_{0}$ (figure $8 \mathrm{~b}, 1.97 \leq B_{0} \leq 2.17 \mathrm{~T}$, discharges \#43957-43960). With every Doppler reflectometry single profile measurement (approx. 15 per shot) is associated one point that represents the sign and magnitude of the maximum value of $\left\langle f_{\text {Doppler }}\right\rangle$ reached in the profile (where $\left\langle f_{\text {Doppler }}\right\rangle$ is the expected value of the asymmetric part of the logarithmic Doppler spectra, defined in section 1.2): this allows to locate the $V_{\perp}$ reversal region in parameter space. A boundary can be sketched at low magnetic field $1.90 \leq B_{0} \leq 1.93 \mathrm{~T}$, which is shifted towards higher densities when $1.97 \leq B_{0} \leq 2.17 \mathrm{~T}$. 

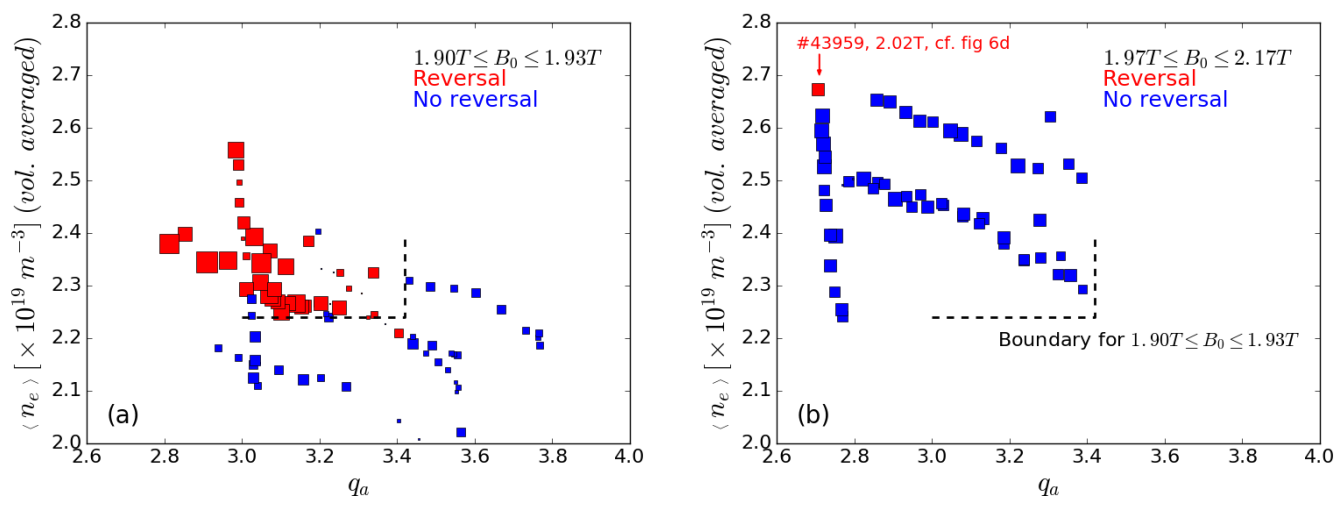

Figure 8: Detections of $V_{\perp}$ reversals in the $\left(q_{a},\left\langle n_{e}\right\rangle\right)$ plane, for the recorded Doppler reflectometry profiles. Measurements are marked according to the maximum value obtained of $\left\langle f_{\text {Doppler }}\right\rangle$ for each profile: red if $\max \left\langle f_{\text {Doppler }}\right\rangle>0$, blue otherwise, and the marker size its proportional to $\left|\max \left\langle f_{\text {Doppler }}\right\rangle\right|$. (a) Shots with $1.90 \leq B_{0} \leq 1.93 T$ (\#43410-13, 43950-52). (b) Shots with $1.97 \leq B_{0} \leq 2.17 T$ (\#43957-43960). The boundary for the $V_{\perp}$ reversal observation is shifted at higher $B_{0}$.

Moreover, it should be noted that the non-observation of the $V_{\perp}$ reversal at lower densities or at higher magnetic fields (\#43952 and 43959, \#43957-60) is not due to a lack of measurements in the $r / a<0.5$ region. For example, figure 9 shows that in the case of discharges \#43959 and \#43960, the reversal or its progressive appearance are located within the accessible zone.

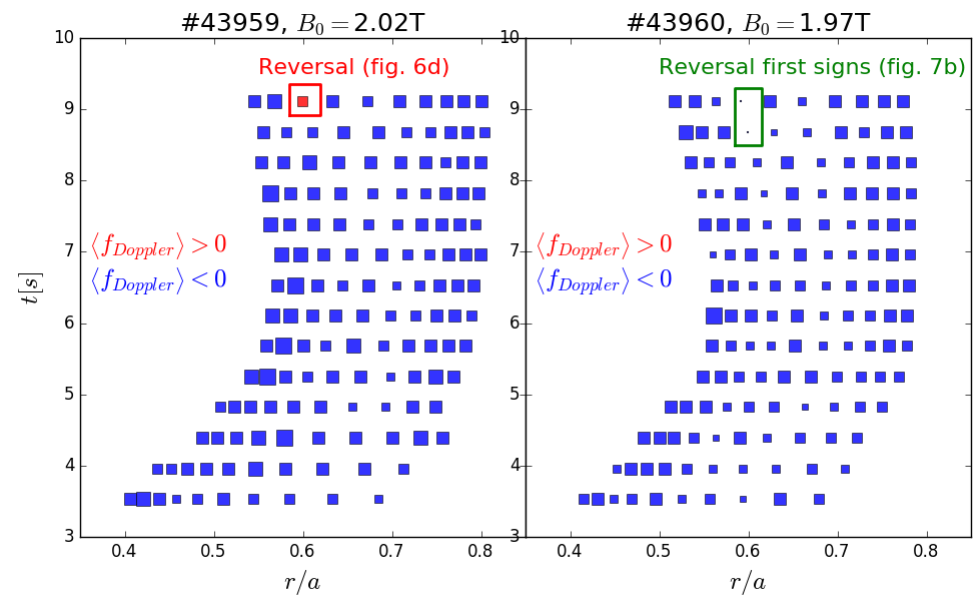

Figure 9: Location of Doppler reflectometry measurements for \#43959 (density ramp at 2.02 T) and \#43960 $\left(q_{a}\right.$ decreasing ramp at $\left.1.97 T\right)$. The marker size and color depends on $\left\langle f_{\text {Doppler }}\right\rangle$ as in figure 8-except that every radial measurements are plotted. For \#43960, the box indicates spectra with a slight appearance of the positively shifted component (cf. blue spectrum in figure $7 b$ ). The $V_{\perp}$ reversal develops within the accessible region.

In conclusion, the influence of the edge safety factor, the electron density, and the vacuum magnetic field on the perpendicular velocity reversal has been investigated. From a reference state $\left(q_{a} \simeq 3, n_{l} \simeq 3.5 \times 10^{19} \mathrm{~m}^{-2}\right.$, 
$B_{0} \simeq 1.9 \mathrm{~T}$, non-circular cross-section) where the reversal is detected, small relative variations of these parameters could affect this phenomenon. The $V_{\perp}$ reversal was observed to be favoured by increasing electron density, decreasing the vacuum magnetic field or decreasing the edge safety factor to values close to 3 . We also note that it is likely that a non-circular plasma shape is also a requirement for the $V_{\perp}$ reversal. In effect, it has not been observed in plasmas with circular cross-section. However, such an influence cannot be concluded firmly, due to a lack of comparable plasma conditions (for example the discharge \#43961- shown in figure $2 \mathrm{~b}$ - had a decreasing $q_{a} \operatorname{ramp}$ up to $q_{a, \text { min }} \simeq 3.15$, which remains higher than the $q_{a, \text { min }} \simeq 2.8$ reached in shot \#43413).

\section{Investigation on the possible mechanism causing the perpendicu- lar velocity reversal}

This section presents additional measurements aiming at studying the mechanisms that could cause this local change of plasma perpendicular flow. Because of the low $q_{a}$ value and the absence of strong sources of fast particles (plasmas being ohmic), MHD appears as a good candidate to explain these observations.

A correlation between the magnetics measurements during $q_{a}$ ramps and the perpendicular velocity reversal has been noticed: a bump in the MHD fluctuations appears at the time of the reversal.

The MHD activity signal $s_{M H D}$ is the RMS of $B_{\theta}$ fluctuations (with a frequency below $10 \mathrm{kHz}$ ) measured by 23 Mirnov coils at a constant toroidal angle and 10 coils at constant poloidal angle [15]. During the series of decreasing $q_{a}$ ramps, the MHD signal showed a reproducible behaviour, shown on figure 10 for discharge \#43413. A characteristic "bump" can be noted between $q_{a} \sim 3.4$ and $q_{a} \sim 3.1$, before the crossing of the LCFS by the $q=3$ surface.

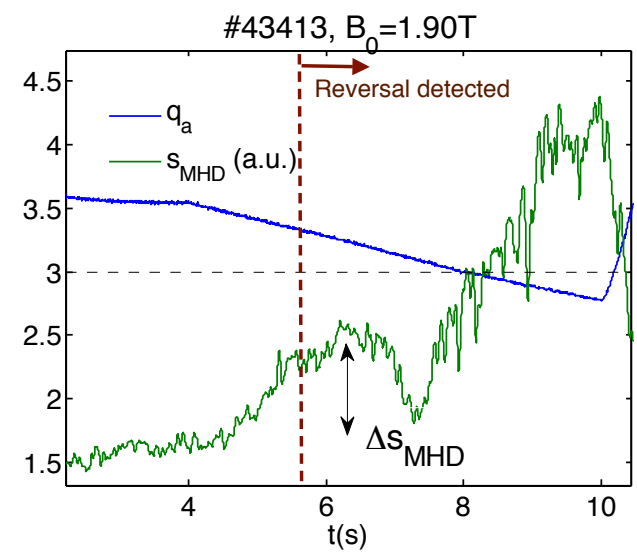

Figure 10: Time evolution of $q_{a}$ and $M H D$ activity signal $s_{M H D}$ during discharge \#43413. The time from which the $V_{\perp}$ reversal is (gradually) detected is indicated. 

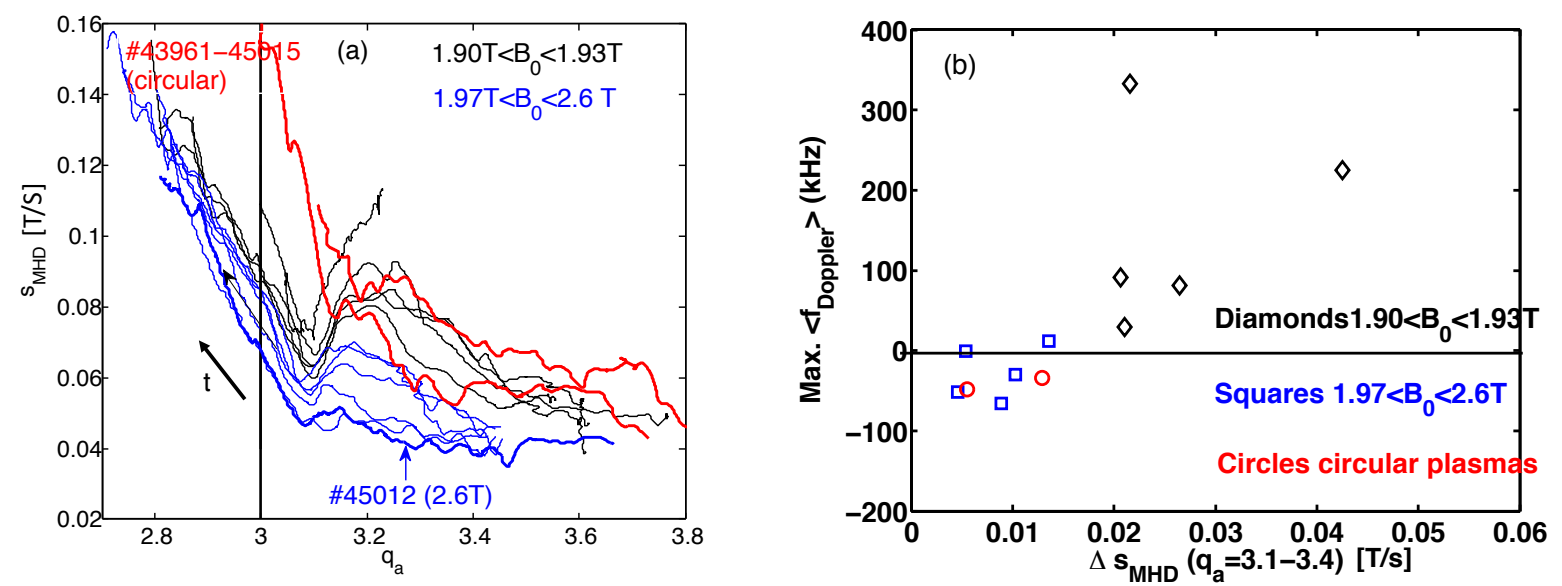

Figure 11: (a) Temporal evolution of discharges with a $q_{a}$ ramp, described as curves in the $\left(q_{a}, s_{M H D}\right)$ plane with time as parameter. Discharges with circular plasma are in red, with non-circular cross section in blue (for $1.97<B_{0}<2.6 \mathrm{~T}$ ) or in black (for $1.90<B_{0}<1.93 \mathrm{~T}$ ). A larger "bump" of MHD activity $s_{M H D}$ can be seen for discharges with lower $B_{0}$ when $q_{a} \sim 3.1-3.4$. The shot 45012 (2.6 T), for which additional measurements will be shown in figure 13, is represented in bold blue. (b) Same discharges (with the same color code), are plotted in the plane formed with the two parameters - defined in the text - quantifying the magnetic activity "bump" $\left(\triangle s_{M H D}\right)$ and the most positive Doppler shift obtained $\left(\max \left\langle f_{\text {Doppler }}\right\rangle\right)$.

This transient episod of MHD activity before reaching $q_{a}=3$ appears to be related with the $V_{\perp}$ reversal. The figure 11a plots the evolution of all discharges with a decreasing $q_{a}$ ramp as curves in the $\left(q_{a}, s_{M H D}\right)$ plane. As mentionned before, the $V_{\perp}$ reversal is clearly observed for the lowest values of the magnetic field $B_{0}<1.93 \mathrm{~T}$, and with non-circular plasma cross-section. Distinct qualitative evolutions of the MHD activity during the $q_{a}$ ramp are noticed: this transient "bump" is present for the discharges with $B_{0}<1.93 \mathrm{~T}$ (black lines in figure 11a), but is attenuated at larger magnetic fields $1.97<B_{0}<2.6 \mathrm{~T}$. MHD activity evolution is qualitatively different for the two circular plasmas with $B_{0}=1.90 \mathrm{~T}$ and $B_{0}=1.97 \mathrm{~T}$.

The figure $11 \mathrm{~b}$ attempts to put in evidence the correlation between the $V_{\perp}$ reversal and the episod of MHD before $q_{a}=3$. It plots, for the each of these discharges, the maximum value of $\max \left\langle f_{\text {Doppler }}\right\rangle$ (defined in in section 1.2) obtained.

It is shown that the discharges with a significant bump of MHD activity when $q_{a} \sim 3.1-3.4$ are precisely the discharges with a perpendicular velocity reversal. The mechanism explaining this correlation remains to be characterized. A possibility would be that an energy transfer occur when magnetic fluctuations are damped, resulting in a strengthening of the perpendicular flow shearing.

During discharges with a decreasing $q_{a}$ ramp, fast magnetic measurements at different stages of the plasma ramp have been made. A spectrogram of mode frequency is shown on figure 12 for discharge \#43413. The evolution of mode frequency is similar for all the discharges with a $q_{a}$ ramp, regardless of the magnetic field value (and the observation or not of a $V_{\perp}$ reversal). A mode is detected in the frequency range $2-2.5 \mathrm{kHz}$. 


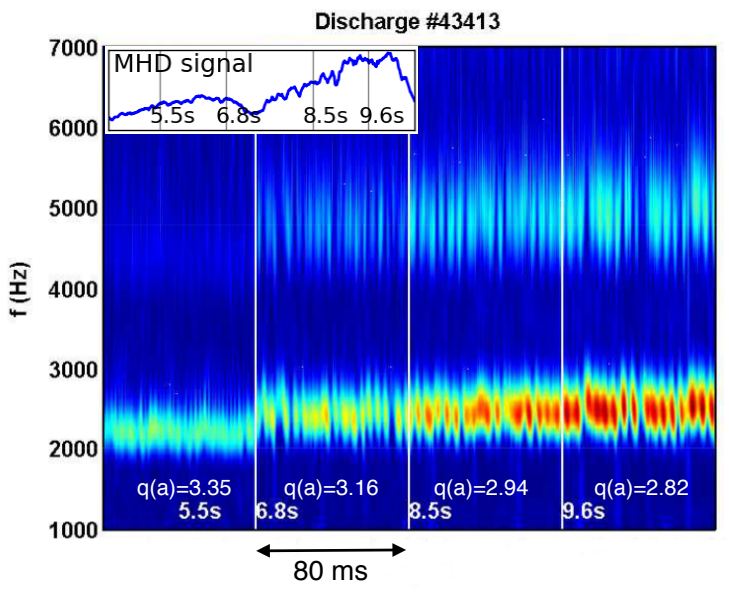

Figure 12: Fast magnetics spectrograms at four different stages of the decreasing $q_{a}$ ramp for discharge \#43413.

In an additional discharge at a higher magnetic field $B_{0}=2.6 \mathrm{~T}$ (\#45012, described in table 2), the correlation ECE [16] observes the progressive appearance of a $2 \mathrm{kHz}$ mode during the decreasing $q_{a}$ ramp (these measurements are done in O-mode first harmonic, on the high field side). This is presented in figures 13c-f. The magnetic island is centered at $r / a \simeq 0.75$ - close to the $q=2$ surface according to EFIT calculations - and its width grows up to approximately $10 \mathrm{~cm}$. Figures 13a-b shows that MHD activity in this discharge shares some common characteristics with the discharges having a $V_{\perp}$ reversal: light bump when $q_{a} \sim 3.2-3.4$, and the same $2 \mathrm{kHz}$ mode detected by Mirnov coils. This allows the identification of the Mirnov fluctuations observed in all discharges as a $m / n=2 / 1$ mode, even if correlation ECE measurements cannot access the $q=2$ region at the magnetic fields of the $V_{\perp}$ reversal. In effect, at $B_{0}=1.9 \mathrm{~T}$, correlation ECE measurements in $\mathrm{X}$-mode second harmonic were restricted to the plasma centre, where they detect large sawtooth precursor oscillations progressively developing during the $q_{a}$ decreasing ramp - however this is not necessarily related to the $V_{\perp}$ reversal.

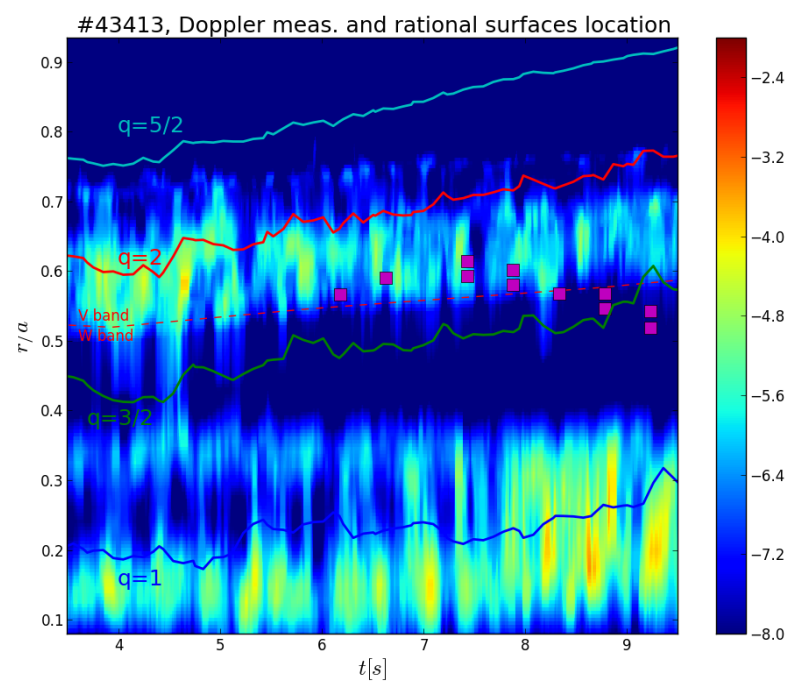

Figure 14: Normalized radii of the $V_{\perp}$ reversal (squares), and time-of-flight jumps for discharge \#43413. The color map is in arbitrary logarithmic units. Rational surfaces position estimated by CRONOS, and the location of the $V / W$ band frequency boundary (in red dashed lines) for the reflectometry are also displayed. 

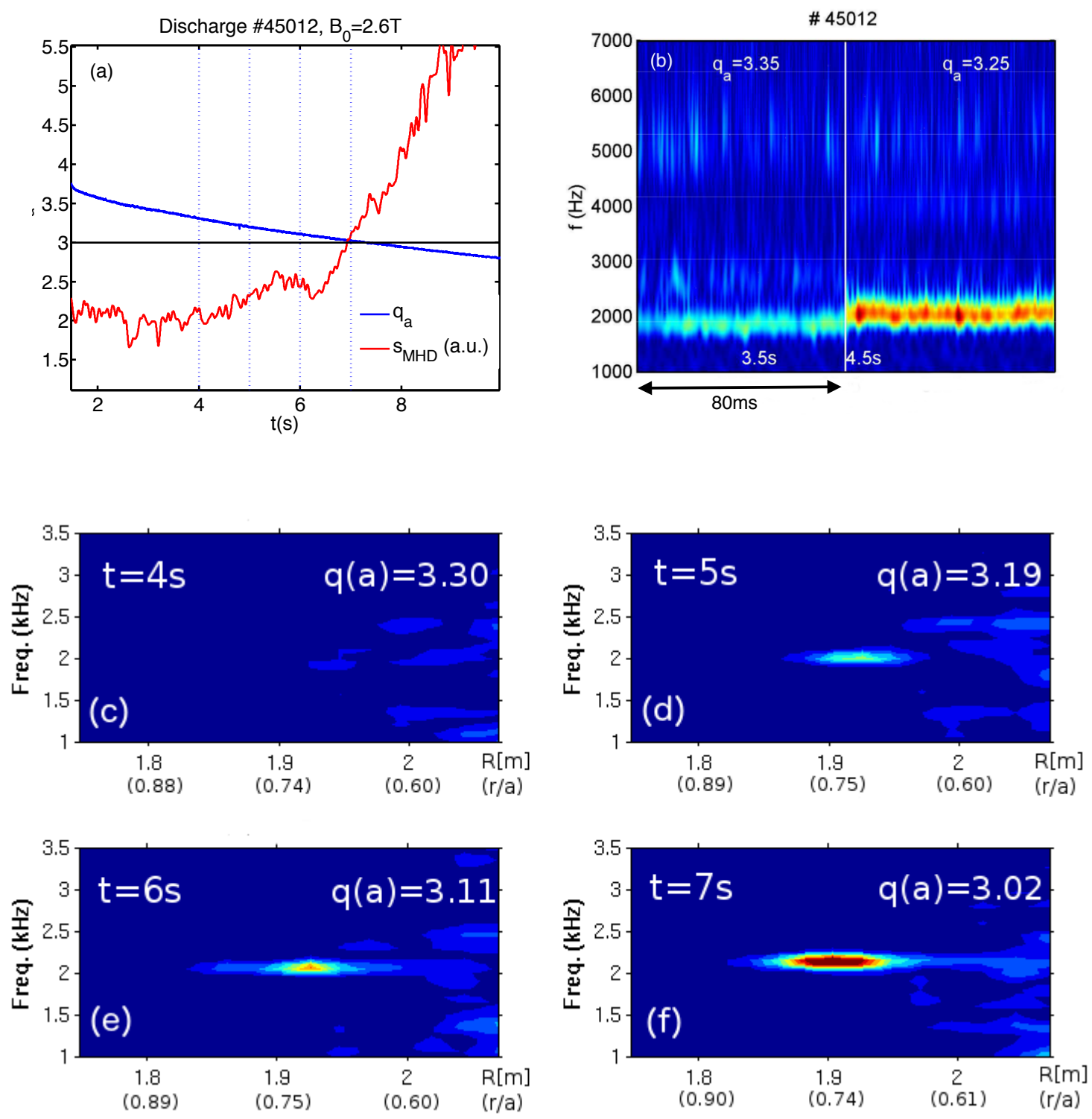

Figure 13: (a) Time evolution of $q_{a}$ and magnetic fluctuations RMS during discharge \#45012. Time of ECE fast acquisition are represented by the vertical lines. (b) Magnetic fluctuations spectrogram at the beginning of discharge \#45012 (at $B_{0}=2.6 \mathrm{~T}$ ). (c-f) Electron temperature fluctuations spectrogram from correlation ECE (O mode 1st harmonic, high field side), at the times indicated in subfigure a, and as a function of the major (or normalized) radius. 
Finally, an attempt to evaluate the localization of the reversal relatively to the low order rational surfaces is shown in figure 14, which maps together for the shot \#43413: (1) the radii where the $V_{\perp}$ reversal is observed, (2) the main rational surfaces position calculated with CRONOS [17], and (3) the amplitude of time-of-flight jumps detected by reflectometry, indicative of the possible presence of large magnetic islands. The following remarks can be formulated:

For (1), the Doppler reflectometry measurements points (squares) displayed are those with a spectra dominated by the $V_{\perp}>0$ component. The apparent inner motion for $t>7 \mathrm{~s}$, which follows the variations of the incident beam poloidal launching angle (cf figure 2a), may be artificially due to uncertainities in the density profile reconstruction that affects the beam tracing calculations.

(2) The modelling code CRONOS has been used to evaluate the rational surface locations (with significant error bars, in the absence of MSE measurements and of a full $T_{e}$ profile).

(3) The V and W-band Tore Supra reflectometers $[18,19,20]$ can detect large magnetic islands (typically $\mathrm{m}, \mathrm{n}=1,2,3)$ through jumps visible on the measured time of flight due to the associated flattening of the density profile [21]. In this series of experiments, reflectometry cannot access to the plasma edge, and therefore the density profile cannot be evaluated (this is usually done by successive integration, using the edge density as a boundary condition). Here the location of the cut-off layer has been estimated with the same beam tracing code (and density profiles) as for Doppler reflectometry.

In spite of these uncertainities, it can be shown that the reversal occurs in the neighbouring region of the $q=2$ and $q=3 / 2$ surfaces. Moreover, the reflectometry confirms the observation of a mode in the neighbouring of $q=2$ detected by ECE.

\section{Discussion and summary}

A reversal of the perpendicular velocity cannot be explained by ripple-induced transport [9], that usually determines $V_{\perp}$ in the plasma region of interest in Tore Supra [6,22], because this mechanism would only induce a negative $E_{r}$. This reveals the presence of another mechanism, related with MHD activity.

The context of these observations could favour a coupling between the $(3,1)$ and the $(2,1)$ modes. In effect, during a decreasing $q_{a}$ ramp, the $q=3$ surface is close to the edge: in numerical simulations [23] this has been shown to strongly increase the $(2,1)$ tearing stability index. Moreover, while this surface approaches the edge, its perpendicular velocity undergoes large changes: even if no edge measurements exist for these discharges, typical values for edge $V_{\perp}$ in L-mode are (see e.g. Hennequin et al. [11]) from $-5 \mathrm{~km} / \mathrm{s}$ at $r / a=0.95$ to 0 at the LCFS. It is therefore possible that a coincidence between the frequencies of the $(3,1)$ and the $(2,1)$ modes occurs during the $q_{a}$ ramp.

Numerical simulations using a two-fluid model [24] have shown that a helical perturbation can induce plasma rotation in the ion diamagnetic direction (corresponding to the positives $E_{r}$ and $V_{\perp}$ observed during the reversal). Such an effect is also predicted for small perturbations that are below the field penetration threshold. It could be at the origin of a rotation change induced by the $(3,1)$ mode on the $(2,1)$ surface. Another explanation accounting for the positive $E_{r}$ would be the enhanced electron transport due to stochasticization of magnetic field lines [25].

The $E_{r}$ reversal is favoured at small magnetic fields and large densities, in the explored parameter space. This would suggest a sensitivity to $\beta$, with a threshold-like triggering (given the strong impact of small variations of these parameters) when $\beta$ exceeds a critical value. High $\beta$ tends to raise the threshold in magnetic perturbation amplitude for mode locking [24, 26]. It could also increase the diamagnetic electron frequency, proportional to $\nabla p_{e} / B$, and hence the torque that pushes the plasma rotation towards the ion diamagnetic direction (e.g. see equations 9 and 10 in $\mathrm{Yu}$ et al. [24]).

The influence of induced magnetic perturbations on plasma rotation has been well documented, for example in COMPASS-C [27], Tore Supra [28], LHD [29], TEXTOR [30], TUMAN 3-M [31, 32], ASDEX Upgrade [33], or TJ-II [34, 35]. However, one interest of the observations presented here is that a spontaneous reversal of the perpendicular velocity is observed, with a parameter dependence that indicates a threshold effect. It would be interesting to investigate if a common mechanism could relate these observations to other classes of experimental results, like the toroidal rotation reversals.

Such reversals of toroidal rotation have been observed in TCV [36, 37], Alcator C-Mod [38], ASDEX Upgrade [39, 40], and later proved to be related with SOC-LOC transitions [41]. An important difference is that the toroidal velocity reversal affects a major part of the plasma, whereas in Tore Supra the observed 
$E_{r}$ reversal remains localized at $r / a \sim 0.5-0.6$. However, some common features can be noticed, especially when the plasma configuration is close to Tore Supra (an extensive study has been done by Duval et al. [37]): in L-mode limited plasmas, the TCV reversal has the same sensitivity to density and magnetic field (shift towards positive $E_{r}$ at high densities), occurs in plasmas where the edge safety factor is close to or below 3 . A 2/1 mode has also been suspected to be involved in the mechanism [37], even if other possible explanations are related with ITG/TEM transitions.

Another interesting question would be whether the mechanism causing the $V_{\perp}$ reversal, able to induce a strongly sheared perpendicular flow, could be involved in the triggering of ITBs which are known to appear in the vicinity of low order rational magnetic surfaces [42, 43].

To sum up, in Tore Supra L-mode limited ohmic plasmas, $V_{\perp}$ measured by Doppler reflectometry was observed to reverse in a localized zone close to a normalized radius $\sim 0.5-0.6$, changing from a negative value (corresponding to a negative radial electric field $\left.E_{r}\right)$ to a positive value $\left(E_{r}>0\right)$. Plasma parameters have been varied around the point of observation, showing that this reversal is favoured by a small decrease in the magnetic field, or a small increase in density: this indicates a threshold-like dependence in $\beta$. The $V_{\perp}$ reversal is related with a characteristic "bump" in the the MHD fluctuation RMS signal, whose amplitude decrease during a ramp-down of the edge safety factor as it approaches $q_{a} \sim 3.1-3.2$. A $m / n=2 / 1$ mode could play a role in this change in the perpendicular flow.

\section{Acknowledgements}

We would like to thank Dr. Q. Yu for fruitful discussions. Pr. U. Stroth and Dr. H. Weisen are thanked for reviewing the $\mathrm{PhD}$ thesis associated with this work. This work was carried out within the framework of the European Fusion Development Agreement and the French Research Federation for Fusion Studies (FR-FCM). It was supported by the European Communities under the contract of Association between Euratom and CEA. The views and opinions expressed herein do not necessarily reflect those of the European Commission. Financial support was also received from Agence Nationale de la Recherche under contract ANR-06-BLAN-0084.

\section{References}

[1] Biglari H., Diamond P. and Terry P., 1990 Phys. Fluids B 21

[2] Waltz R.E., Kerbel G.D. and Milovich J., 1994 Phys. Plasmas 12229

[3] Terry P.W., 2000 Rev. Mod. Phys. 72109

[4] Itoh K. and Itoh S.I., 1996 Plasma Phys. Control. Fusion 381

[5] Rutherford P.H., 1970 Phys. Fluids 13482

[6] Trier E., Eriksson L.G., Hennequin P., Fenzi C., Bourdelle C., Falchetto G., Garbet X., Aniel T., Clairet F. and Sabot R., 2008 Nucl. Fusion 4892001

[7] Connor J.W. and Hastie R.J., 1973 Nucl. Fusion 13221

[8] Boozer A.H., 1980 Phys. Fluids 232283

[9] Garbet X. et al., 2010 Phys. Plasmas 171

[10] Hennequin P., Honoré C., Truc A., Quéméneur A., Lemoine N., Chareau J.M. and Sabot R., 2004 Rev. Sci. Instrum. 753881

[11] Hennequin P., Honoré C., Truc A., Quéméneur A., Fenzi-Bonizec C., Bourdelle C., Garbet X., Hoang G. and the Tore Supra team, 2006 Nucl. Fusion 46 S771

[12] Bourdelle C., Garbet X., Hoang G.T., Ongena J. and Budny R.V., 2002 Nucl. Fusion 42892 
[13] Vermare L., Hennequin P., Gürcan O.D., Bourdelle C., Clairet F., Garbet X. and Sabot R., 2011 Phys. Plasmas 18

[14] Conway G.D., Schirmer J., Klenge S., Suttrop W., Holzhauer E. and the ASDEX Upgrade team, 2004 Plasma Phys. Control. Fusion 46951

[15] Moreau P., Defrasne P., Joffrin E., Laurent F.S. and Martin G., 2003 Rev. Sci. Instrum. 744324

[16] Ségui J.L., Molina D., Giruzzi G., Goniche M., Huysmans G., Maget P., Ottaviani M. and the Tore Supra team, 2005 Rev. Sci. Instrum. 76123501

[17] Artaud J. et al., 2010 Nucl. Fusion 50043001

[18] Clairet F., Bottereau C., Chareau J.M. and Sabot R., 2003 Rev. Sci. Instrum. 741481

[19] Sabot R. et al., 2006 Plasma Phys. Control. Fusion 48 B421

[20] Gil C. et al., 2009 Fusion Sci. Technol. 561219

[21] Vermare L., Clairet F., Heuraux S. and Leclert G., 2005 Plasma Phys. Control. Fusion 471895

[22] Fenzi C. et al., 2011 Nucl. Fusion 51103038

[23] Fitzpatrick R., Hastie R., Martin T. and Roach C., 1993 Nucl. Fusion 331533

[24] Yu Q., Günter S. and Finken K.H., 2009 Phys. Plasmas 16042301

[25] Rechester A.B. and Rosenbluth M.N., 1978 Phys. Rev. Lett. 4038

[26] Fitzpatrick R., 2012 Plasma Phys. Control. Fusion 54094002

[27] Hender T. et al., 1992 Nucl. Fusion 322091

[28] Payan J. et al., 1995 Nucl. Fusion 351357

[29] Ida K. et al., 2002 Phys. Rev. Lett. 88015002

[30] Finken K.H. et al., 2005 Phys. Rev. Lett. 941

[31] Askinazi L.G., Golant V.E., Kornev V.A., Lebedev S.V., Tukachinsky A.S., Vildjunas M.I. and Zhubr N.A., 2006 Plasma Phys. Control. Fusion 48 A85

[32] Bulanin V.V., Askinazi L.G., Lebedev S.V., Gorohov M.V., Kornev V.A., Petrov A.V., Tukachinsky A.S. and Vildjunas M.I., 2006 Plasma Phys. Control. Fusion 48 A101

[33] Conway G.D., Fietz S., Müller H.W., Lunt T., Simon P., Suttrop W., Maraschek M., Happel T. and Viezzer E., 2015 Plasma Phys. Control. Fusion 57014035

[34] Bondarenko O., Estrada T., Jiménez-Gómez R., López-Bruna D., Happel T., Romero J., López-Fraguas A., Ascasíbar E. and Blanco E., 2010 Contrib. to Plasma Phys. 50605

[35] Estrada T., Ascasíbar E., Blanco E., Cappa A., Hidalgo C., Ida K., López-Fraguas A. and van Milligen B.P., 2016 Nucl. Fusion 56026011

[36] Bortolon A., Duval B.P., Pochelon A. and Scarabosio A., 2006 Phys. Rev. Lett. 971

[37] Duval B.P., Bortolon A., Karpushov A., Pitts R.A., Pochelon A., Sauter O., Scarabosio A. and Turri G., 2008 Phys. Plasmas 151

[38] Rice J.E., Ince-Cushman A.C., Reinke M.L., Podpaly Y., Greenwald M.J., LaBombard B. and Marmar E.S., 2008 Plasma Phys. Control. Fusion 50124042

[39] Angioni C. et al., 2011 Phys. Rev. Lett. 1071 
[40] McDermott R.M., Angioni C., Conway G.D., Dux R., Fable E., Fischer R., Pütterich T., Ryter F., Viezzer E. and the ASDEX Upgrade team, 2014 Nucl. Fusion 54043009

[41] Rice J. et al., 2013 Nucl. Fusion 53033004

[42] Joffrin E., Challis C.D., Hender T.C., Howell D.F. and Huysmans G.T.A., 2002 Nucl. Fusion 42235

[43] Austin M.E. et al., 2006 Phys. Plasmas 13082502 